\title{
Development and evaluation of collision warning/collision avoidance algorithms using an errable driver model
}

\author{
Hsin-Hsiang Yang* and Huei Peng \\ Lay Automotive Laboratory, Department of Mechanical Engineering, University of Michigan, \\ Ann Arbor, MI 48105, USA
}

(Received 15 April 2010; final version received 9 August 2010)

\begin{abstract}
Collision warning/collision avoidance (CW/CA) systems must be designed to work seamlessly with a human driver, providing warning or control actions when the driver's response (or lack of) is deemed inappropriate. The effectiveness of $\mathrm{CW} / \mathrm{CA}$ systems working with a human driver needs to be evaluated thoroughly because of legal/liability and other (e.g. traffic flow) concerns. CW/CA systems tuned only under open-loop manoeuvres were frequently found to work unsatisfactorily with human-in-theloop. However, tuning CW/CA systems with human drivers co-existing is slow and non-repeatable. Driver models, if constructed and used properly, can capture human/control interactions and accelerate the $\mathrm{CW} / \mathrm{CA}$ development process. Design and evaluation methods for $\mathrm{CW} / \mathrm{CA}$ algorithms can be categorised into three approaches, scenario-based, performance-based and human-centred. The strength and weakness of these approaches were discussed in this paper and a humanised errable driver model was introduced to improve the developing process. The errable driver model used in this paper is a model that emulates human driver's functions and can generate both nominal (error-free) and devious (with error) behaviours. The car-following data used for developing and validating the model were obtained from a large-scale naturalistic driving database. Three error-inducing behaviours were introduced: human perceptual limitation, time delay and distraction. By including these error-inducing behaviours, rear-end collisions with a lead vehicle were found to occur at a probability similar to traffic accident statistics in the USA. This driver model is then used to evaluate the performance of several existing $\mathrm{CW} / \mathrm{CA}$ algorithms. Finally, a new $\mathrm{CW} / \mathrm{CA}$ algorithm was developed based on this errable driver model.
\end{abstract}

Keywords: active safety; driver model; driver error; CW/CA algorithm

\section{Introduction}

Driver errors are known to be responsible for a large number of traffic accidents. Among all the accidents, collision with another motor vehicle was the most common first harmful event for crashes [1]. As early as the 1950s, collision warning algorithms and devices were developed for aviation applications [2,3]. Ground vehicle CW/CA problems were discussed by Gibson and Crooks [4]. In his paper, Gibson described the concept of safe travel. In the 1980s, the

\footnotetext{
*Corresponding author. Email: seanyang@umich.edu

ISSN 0042-3114 print/ISSN 1744-5159 online

(C) 2010 Taylor \& Francis

DOI: $10.1080 / 00423114.2010 .515745$

http://www.informaworld.com
} 
Ministry of Construction in Japan organised a committee to focus on the study of advanced vehicle technologies, including obstacle detecting and collision avoidance [5]. Early collision warning algorithms were developed and introduced in [6,7]. In the early 1990s, as part of the push for intelligent vehicle highway system, collision warning/avoidance systems were widely studied [8-10].

In the 1990s, Burgett et al. [11] proposed a rear-end collision warning algorithm. The driving situations were divided into three zones. The boundary between the zones and warning criteria were defined accordingly. Kiefer et al. [12] reported minimum elements required for the development of forward collision warning systems. Crash alert timing was studied by conducting human factor analysis. Doi et al. [13] developed an algorithm based on kinematic relationship between two vehicles and addressed collision problem in both straight and curved roads. Brunson et al. [14] refined the algorithm developed by Burgett et al. at NHTSA. Their algorithm used current vehicle states and assumed driver reaction time to calculate a range which they named 'miss-distance'. When the miss-distance is smaller than a threshold, an alert would be issued. Zhang and Antonsson [15] gave another interpretation of Brunson and NHTSA's algorithm. Instead of calculating the miss-distance, they used the vehicle range to calculate a reaction time and called it the 'time to last second braking'. They claimed that this new approach has better agreement with human judgement and directly quantifies the threat level of the driving situation. All the above algorithms are based on assumed kinematic equations. By measuring engineering variables such as range, speed and acceleration, the severity of collision threats is assessed and is used as the basis for warning or control.

An alternative approach was using time to collision (TTC) as the warning criteria. As suggested by Gibson [16] and Lee [17], human drivers rely more on the TTC for collision judgement than other signals such as range or time headway. Lee and Peng [18] provided a systematic method for evaluating collision warning algorithms and they claimed that TTC is a better metric for collision threat than other kinematic variables. Using TTC and lead vehicle acceleration would further improve the performance in terms of warning precision and true positive rate. Hirst and Graham [19] suggested a TTC algorithm with speed dependency. Miler and Huang [20] added an extra term to the TTC algorithm. This extra term includes human reaction time, reduced speed and estimated road friction coefficient. The reaction time modelled human's time delay and the reduced speed and the estimated road coefficient can be adjusted depending on the severity of the situation.

Collision warning/collision avoidance (CW/CA) systems are driver-assistant systems for situations when the driver is not able to avoid or mitigate a crash; in other words, when the driver is either making a mistake or is not able to handle the situation. Therefore, to evaluate $\mathrm{CW} / \mathrm{CA}$ algorithms, models that achieve driving tasks perfectly are not very useful. On the contrary, a model that makes mistakes similar to human drivers is more useful for the development of CW/CA systems. In the following sections, an errable driver model will be introduced. A stochastic driver model developed based on a large quantity of realistic driving data is used as the basis of this errable driver model. Three error mechanisms are used to create an 'errable' model (i.e. the model makes mistakes in a manipulated fashion). Subsequently, several conventional evaluation methods for $\mathrm{CW} / \mathrm{CA}$ algorithms are reviewed and the errable driver model is used to improve those evaluation methods. Moreover, a new evaluation method that fully utilises the strength of the errable driver model is introduced. This method evaluates the timing of $\mathrm{CW} / \mathrm{CA}$ algorithms by comparing them with probabilities of crash predicted by the errable driver model (Figure 1). Finally, a new CW/CA algorithm is developed by utilising the probability of crash as the criteria for issue warning. This algorithm is adaptive and provides different levels of warning. A simple example was shown and comparisons with other $\mathrm{CW} / \mathrm{CA}$ algorithms were presented. 


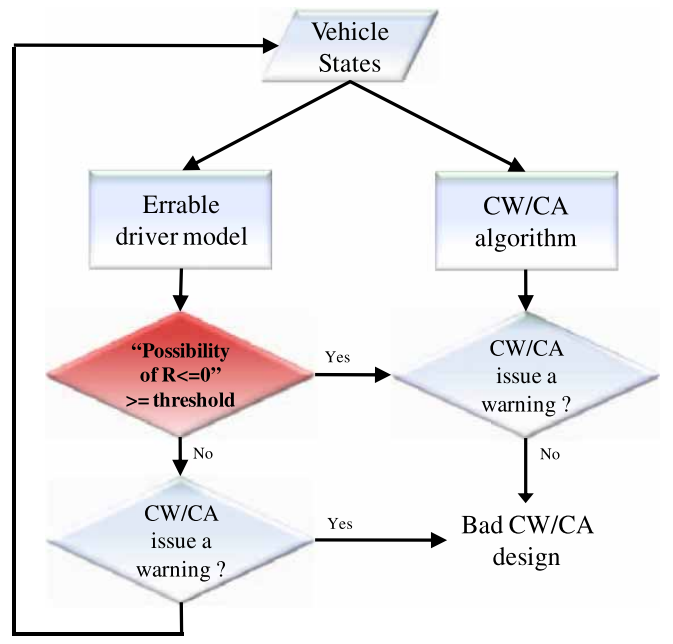

Figure 1. CW/CA evaluation with errable driver model.

\section{Errable driver model}

An errable driver model is a model that normally executes driving tasks (e.g. car-following) properly and could be made to produce devious behaviour (just like human), which could result in accidents or near-accidents that are of interest to designers of active safety systems. Driver errors can be viewed as a recurring event which, in combination with events from surrounding vehicles, could result in an accident. For example, a driver may be distracted or engaged in alternative control tasks and thus fails to adjust vehicle speed at the regular cadence. If the leading vehicle happens to decelerate at the wrong moment, a rear-end collision could occur. The human behaviour (distraction) and lead vehicle deceleration can be described by stochastic processes. If proper human cognition/error mechanisms are included and proper probability functions (from human driving data) are used to introduce human errors, it is possible to reproduce accident/incident behaviour that is statistically similar to field-testing results - which is a major goal of our research. The field-testing database used is from the road-departure crash-warning (RDCW) system field operational test [21]. The RDCW system was designed to analyse road departure behaviour, which was installed on 10 test vehicles. Large quantity of naturalistic field-driving data was collected, which are used for our driver model development.

An errable driver model was developed in a previous work [22] and used here for the evaluation and development of CW/CA algorithms (Figure 2). This model emphasises the stochastic nature of a driver instead of considering driving as a deterministic process. The errable driver model was constructed based on the assumption that a driver normally has a desired target acceleration which can be calculated by a deterministic process, like many other car-following driver models [23-28]. However, the target acceleration will be achieved with some stochastic deviations. The randomness is due to driver imperfection in perception, decision and execution and can be described by a probability distribution function (PDF). The analysis of the RDCW data shows that the mean of this PDF is proportional to the range rate in a function of third-order polynomial and the deviation is a second-order polynomial function of range (1)-(3). Second term in Equation (1) was applied to approximate the human's feedback action for regulating time headway. This additional term is proportional to the difference between the actual range and the desired range. 


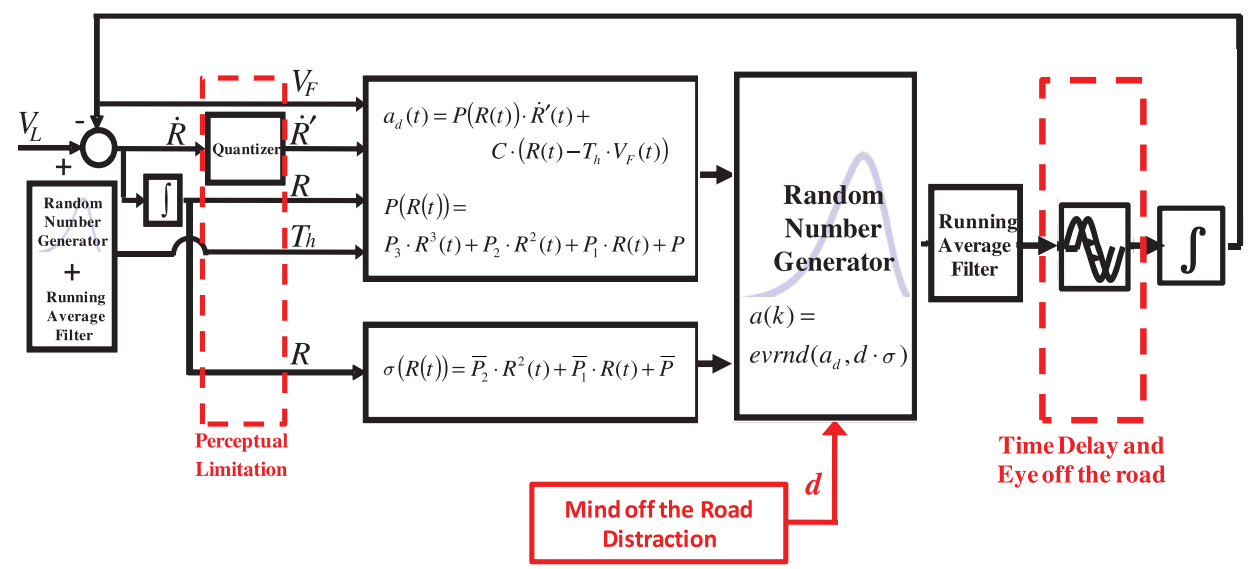

Figure 2. Errable driver model diagram.

The resulting model (1)-(5) is able to simulate the normal driver behaviour and occasional deviations from realistic driving data

$$
\begin{aligned}
a_{\mathrm{d}}(t) & =P(R(t)) \cdot \dot{R}(t)+C \cdot\left(R(t)-T_{\mathrm{h}} \cdot V_{\mathrm{F}}(t)\right) \\
P(R(t)) & =P_{3} \cdot R^{3}(t)+P_{2} \cdot R^{2}(t)+P_{1} \cdot R(t)+P_{0} \\
\sigma(R(t)) & =\bar{P}_{2} \cdot R^{2}(t)+\bar{P}_{1} \cdot R(t)+\bar{P}_{0} \\
a(t) & =f\left(a_{\mathrm{d}}, \sigma\right) \\
f\left(a \mid a_{\mathrm{d}}, \sigma\right) & =\sigma^{-1} \cdot \exp \left(\frac{x-a_{\mathrm{d}}}{\sigma}\right) \cdot \exp \left(-\exp \left(\frac{x-a_{\mathrm{d}}}{\sigma}\right)\right)
\end{aligned}
$$

where $R$ is the range between two vehicles, $P_{i}$ 's the polynomial coefficients, $T_{\mathrm{h}}$ the headway time, $a_{\mathrm{d}}$ the desired acceleration of vehicle, $a$ the implemented acceleration of vehicle and $f$ a random number generator (Table. 1 ).

In an earlier paper by the authors [22], three types of error-inducing behaviours were included: perceptual limitation, time delay and distraction. As explained in [22], these three

Table 1. List of symbols

\begin{tabular}{ll}
\hline Symbol & \multicolumn{1}{c}{ Name } \\
\hline$a_{\mathrm{d}}$ & Driver-desired acceleration \\
$V_{\mathrm{F}}$ & Following vehicle velocity \\
$a_{\mathrm{L}}, V_{\mathrm{L}}$ & Lead vehicle acceleration, velocity \\
$\boldsymbol{R}, \dot{\boldsymbol{R}}$ & Range, range rate \\
$C$ & Desired acceleration gain of range \\
$P$ & Desired acceleration gain of range rate \\
$P_{0}, P_{1}, P_{2}, P_{3}$ & Desired acceleration gain coefficient \\
$\sigma$ & Deviation \\
$\overline{\boldsymbol{P}}_{\mathbf{0}}, \overline{\boldsymbol{P}}_{\mathbf{1}}, \overline{\boldsymbol{P}}_{\mathbf{2}}$ & Deviation gain coefficient \\
$a$ & Implemented acceleration \\
$T_{\mathrm{h}}$ & Time headway \\
$t$ & Time \\
$t_{\text {preview }}$ & Preview time \\
$a_{\text {crash }}$ & Acceleration that leads to a crash \\
\hline
\end{tabular}


error mechanisms can be added to the driver model, and by identifying proper values or PDFs of these error mechanisms, these error-inducing functions could create stochastic behaviours similar to human drivers. The velocity perception limit is a deterministic mechanism and a threshold of 0.1 is used. Time delay of a driver is time-varying and is approximated by a stochastic function. It is approximated as a renewal process, and the PDF of the renewal process is identified through a recursive least square optimisation procedure, by finding the optimal delay time among 20 parallel dynamic processes.

The third and perhaps the most important error-inducing mechanism is driver distraction. Driver is a multi-tasking intelligent subject and needs to or wants to attend to other tasks such as cell phone usage, gawking of traffic incidents or simply day-dreaming. The effect of distraction is modelled as increased standard deviation in control performance (Equation (5)). We analysed the RDCW data and again found the PDF of the alternative renewal process with two independent identical distributions (IID). The IIDs will be used to generate the duration of the normal phase of driving, when the original deviation level in the driver model is used. And then the distracted phase of driving starts, the duration of which is generated from that IID and a higher deviation level will be used. The driver state will then be switched to the normal phase, and the process is repeated until the end of the simulation.

All the three error-inducing behaviours discussed above were implemented, and leading vehicle velocity profiles from actual driving were used as a simulation input. A total of $8,846,976$ miles of driving was simulated and 25 crashes happened (282.6/100M VMT). The probability of rear-end crashes was compared with crash data from NHTSA [1], which has a reported crash rate of 120/100M VMT. Our simulation crash rate is $133 \%$ higher. Owing to the fact that not all minor crashes are reported and drivers might be alerted by passengers or engage unusually high brake actions, i.e. behaviours not adequately captured in the data we used to train the driver model, we think this higher predicted collision rate is reasonable. The fact that the developed driver model is more conservative (result in more crashes), we believe, is beneficial for the evaluation of collision warning/collision avoidance systems.

\section{Evaluating $\mathrm{CW} / \mathrm{CA}$ algorithms}

$\mathrm{CW} / \mathrm{CA}$ algorithms have been studied for more than 50 years. Most of them were designed and evaluated by using a scenario-based method. In this approach, a test matrix was first defined. Then, CW/CA algorithms are exposed to the scenarios within the test matrix and their performance is evaluated. The design and evaluation procedure is illustrated in Figure 3. An example of well-defined test matrices for CW/CA systems can be found in [29-31]. An alternative, a performance-based approach, was proposed by Yang and Yang [32] (Figure 4). A performance boundary was first defined, and then the test matrix was used to search for parameters of the $\mathrm{CW} / \mathrm{CA}$ algorithm that can satisfy the performance requirement. The resulting $\mathrm{CW} / \mathrm{CA}$ algorithm will have guaranteed performance throughout the scenarios covered in the test matrix.

The performance-based design approach still needs pre-determined test scenarios which might not be rich enough. A human-centred approach was developed to compensate for this disadvantage [18]. Significant amount of naturalistic human driving data were analysed and a set of threatening situations were identified. Then, the threatening scenarios were used to test the $\mathrm{CW} / \mathrm{CA}$ algorithms. This human-centred approach benchmarks $\mathrm{CW} / \mathrm{CA}$ algorithm performance by realistic human driving behaviours and provides an optimisation method for obtaining algorithm parameters. Nevertheless, the evaluating data were collected from human driving database without any real crash. In other words, the defined threatening situations may not be severe enough. The errable driver model presented earlier in this paper can address 


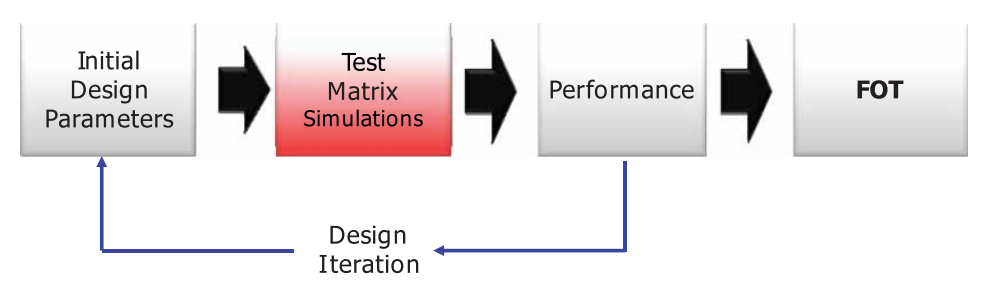

Figure 3. Scenario-test approach design diagram.

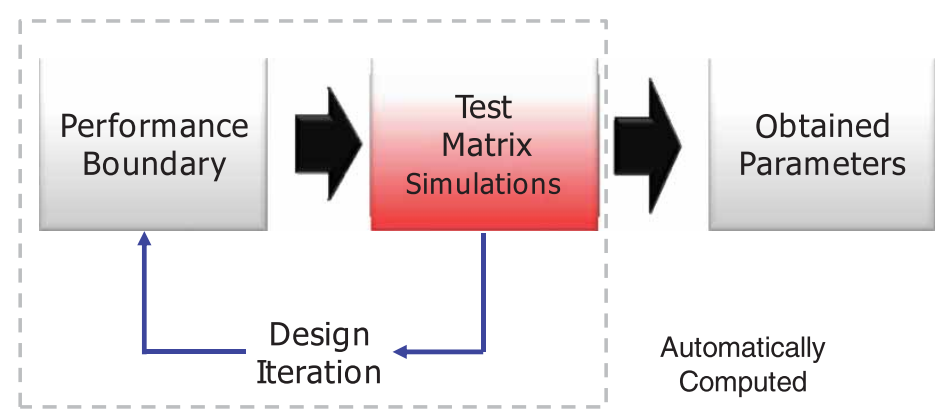

Figure 4. Performance-based scenario-test approach design diagram.

this deficiency. By introducing proper human cognition/error mechanisms, it is possible to generate realistic accidents/incidents. Therefore, the evaluation is human centred and harsh enough to be useful.

The errable driver model can improve the evaluations of $\mathrm{CW} / \mathrm{CA}$ algorithms in several ways. In a scenario-based approach, the driving behaviour of the target vehicle can be replaced by a humanised errable driver model. The errable driver model could generate more realistic driving behaviour than randomly selected scenarios. For a human-centred approach, the errable driver model can be used to generate near-crash or crash manoeuvres. It should be noted that crashes do not happen frequently. Even for large-scale driving database such as [18] with more than 82,000 miles of driving, all the identified threatening situations did not result in actual crash. With the errable driver model, actual crashes can be simulated and used for evaluating $\mathrm{CW} / \mathrm{CA}$ algorithms.

Even though the errable driver model can be used to generate driving behaviour under prescribed test matrices, an alternative is proposed to fully utilise the advantage of the errable driver model and not limited to prescribed test matrices. The errable driver model was developed based on a stochastic driver model and all error-inducing behaviours were imposed as stochastic processes. Given any initial conditions (vehicle velocity, range rate, range and lead vehicle acceleration), the probability of future manoeuvres can be calculated. Hence, the probability of crash can be predicted without exhaustive simulations. A simple example was done by using only the stochastic driver model without any error mechanisms. To demonstrate this concept, a one-step prediction was done. For any given range, range rate and following vehicle speed, the distribution of the acceleration of the following vehicle can be calculated by the stochastic driver model. With the leading vehicle velocity or even acceleration available, the crash accelerations that would end up with a crash in a preview time can be calculated from

$$
R \leq\left(V_{\mathrm{L}}-V_{\mathrm{F}}\right) \cdot t+\frac{1}{2} \cdot\left(a_{\mathrm{L}}-a_{\mathrm{F}}\right) \cdot t_{\text {preview }}^{2} \Longrightarrow a_{\text {crash }} \geq \frac{R-\dot{R} \cdot t_{\text {preview }}}{1 / 2 \cdot t_{\text {preview }}^{2}}+a_{\mathrm{L}}
$$



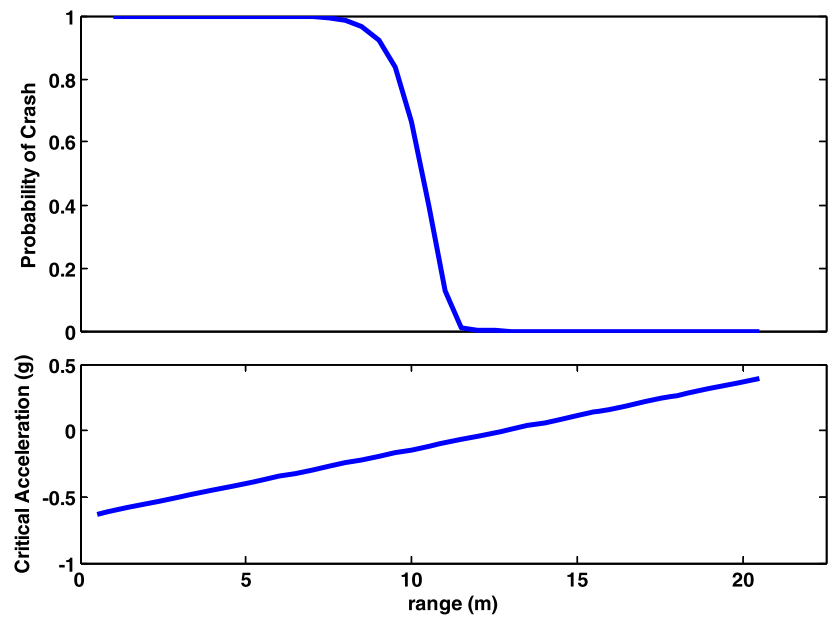

Figure 5. An example of the probability of crash (lead vehicle velocity $=30 \mathrm{~m} / \mathrm{s}$, range rate $=-4 \mathrm{~m} / \mathrm{s}$, lead vehicle acceleration $=-0.22 \mathrm{~g}$ ).

After obtaining the crash accelerations, the probability that the driver will actually accelerate at or beyond those crash accelerations can be calculated by replacing $a_{\mathrm{d}}$ in Equation (5) with

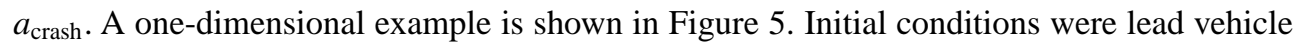
velocity $=30 \mathrm{~m} / \mathrm{s}$, range rate $=-4 \mathrm{~m} / \mathrm{s}$, lead vehicle acceleration $=-0.22 \mathrm{~g}$ and preview time $=2 \mathrm{~s}$. The initial range was varied from 0 to $20 \mathrm{~m}$ and their corresponding probability of crash can be calculated (Figure 5).

Figure 5 can be interpreted as: for the given conditions, whenever the range is smaller than $7.0 \mathrm{~m}$, the target vehicle will have a crash within the next $2 \mathrm{~s}$. And, if the range is large than $12 \mathrm{~m}$, the probability of crash within the next $2 \mathrm{~s}$ is zero. This approach can be further extended to multi-dimensions and compared with the existing CW/CA algorithms. Several algorithms (Table 2) were compared in Figure 6. Some preliminary conclusions can be drawn from Figure 6. For example, Doi's algorithm issues earlier than EDM prediction and Fujita's algorithms are generally late. Those conclusions were consistent with the human-approach evaluation results [34]. All four algorithms are agreed more with EDM prediction in small range rate and differed in large range rate.

Table 2. CW/CA algorithms selected for evaluation.

\begin{tabular}{ll}
\hline & Algorithms \\
\hline Fujita Warning [33] & $R_{\text {warning }}=-\tau \cdot \dot{R}+R_{\min }$ \\
Fujita Braking [33] & $R_{\text {braking }}=\left\{\begin{array}{l}-\tau_{2} \cdot \dot{R}+\tau_{1} \cdot \tau_{2} \cdot \alpha_{1}-0.5 \alpha_{1} \cdot \tau_{1}^{2} \\
\tau_{2} \cdot V_{\mathrm{F}}-0.5 \alpha_{1} \cdot\left(\tau_{2}-\tau_{1}\right)^{2}-\frac{V_{\mathrm{L}}^{2}}{2 \alpha_{2}}\end{array}\right.$ \\
Doi et al. [13] & $R_{\text {warning }}=0.5 \cdot\left(\frac{V_{\mathrm{F}}^{2}}{\alpha_{1}}-\frac{V_{\mathrm{L}}^{2}}{\alpha_{2}}\right)+V_{\mathrm{F}} \cdot \tau_{1}-\dot{R} \cdot \tau_{2}+R_{\text {min }}$ \\
Lee [34] & $\left\{\begin{array}{l}\mathrm{TTI} \leq \mathrm{TTI}_{\text {criterion }} \\
a_{\mathrm{L}} \leq a_{\mathrm{L}_{\text {criterion }}}\end{array}\right.$ \\
\hline
\end{tabular}




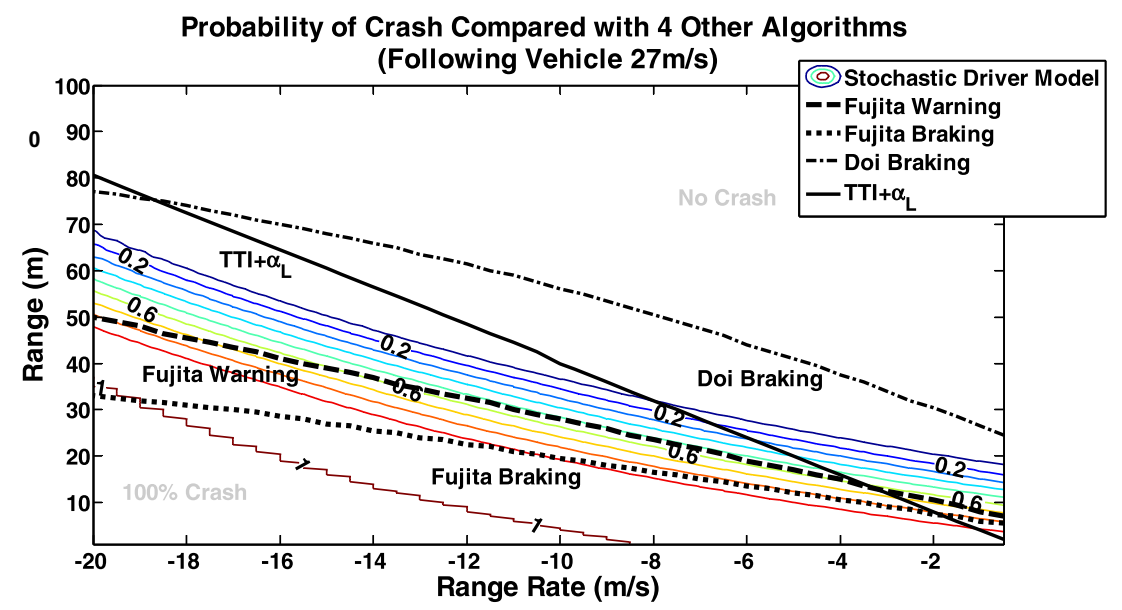

Figure 6. EDM probability of crash compared with other algorithms.

\section{A humanised CW/CA algorithm}

In the previous section, the errable driver model is used to evaluate the $\mathrm{CW} / \mathrm{CA}$ algorithms by comparing the warning timing with the probability of crash. This probability of crash can also be used as the warning criteria. If the predicted probability of crash exceeds a threshold, a warning can be issued. The prediction method provided in the previous section estimates the maximum acceleration which would cause a crash and calculates the probability of crash backwards. This method is computation-efficient but not accurate because it assumes a constant acceleration throughout the whole prediction horizon. A forward multi-step prediction can be used to achieve a higher accuracy. Using current vehicle states as the starting point, the vehicle's possible future states can be predicted with their probability. A simplified example is shown in Figure 7.

The prediction started as state 00 . The next three possible states 10,11 and 12 can be calculated with their probability $P_{00,10}, P_{00,11}$ and $P_{00,12}$, respectively. The prediction can keep going until the preview time $t_{\text {preview }}$ or when the state becomes absorbing. A state is absorbing if the following vehicle speed $V_{\mathrm{F}}$ and/or range rate reaches zero (states 20), i.e. when the vehicle is fully stopped or is slower than the lead vehicle. Meanwhile, the state can also become
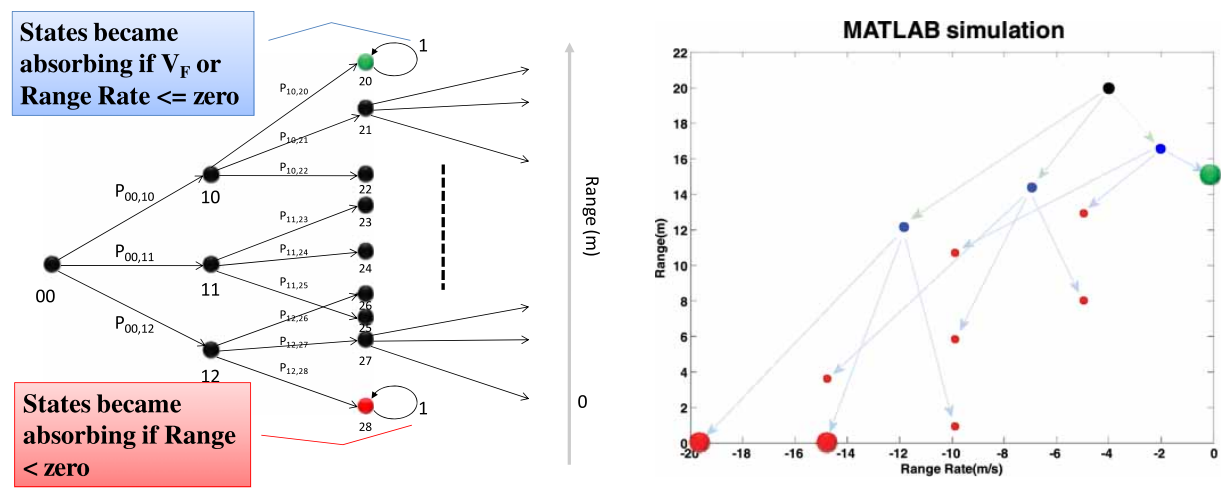

Figure 7. Multi-step prediction. 


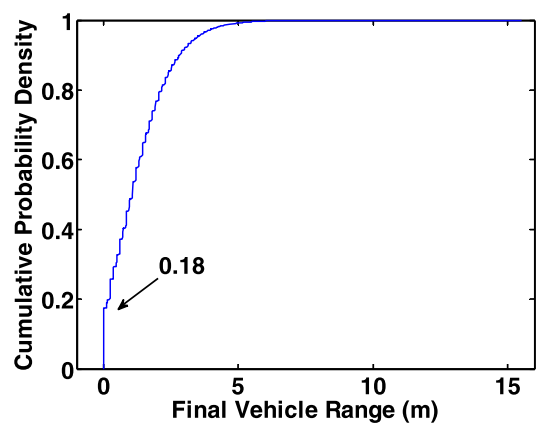

Figure 8. Cumulative probability of vehicle range at the end of the $2 \mathrm{~s}$ preview time.

absorbing if the range is smaller than zero (states 28), which represents a crash. The probability of crash can be calculated by summing up probabilities of all the states that result in crashes. A MATLAB example is shown in Figure 7. The simulation started a black dot (range $20 \mathrm{~m}$ and range rate $-4 \mathrm{~m} / \mathrm{s}$ ). Three possible future vehicle positions are blue dots and final vehicle positions are in red. Two larger red dots represent crashes and a large green dot represents a safe condition that the range rate is equal to zero. Therefore, the possibility that would end up in crashes can be calculated. One simulation result is shown in Figure 8. Initial conditions were $V_{\mathrm{F}}=30 \mathrm{~m} / \mathrm{s}$, range rate $=-4 \mathrm{~m} / \mathrm{s}$, range $=20 \mathrm{~m}, a_{\mathrm{F}}=-0.6 \mathrm{~g}$, preview time $=2 \mathrm{~s}$, prediction states $=12$ and prediction steps $=4$. The probability of a crash in the next $2 \mathrm{~s}$ is $18 \%$ which may be high enough to issue a warning. Figure 9 shows the probabilities of crash for different initial conditions. With error-inducing behaviours and multi-step prediction, probabilities of crash are slightly different from the prediction made in the previous section. The vehicle starts to have a probability of crash earlier because of error-inducing behaviours. However, the probability of crash increases slowly because of the multi-step prediction. The physical interpretation of multi-step prediction means that the model has more steps to correct its behaviour.

This probability of crash distribution (Figure 9) can be used to construct a warning map. One benefit of this algorithm is that the probability of crash can be selected to set different levels of warning. Algorithm developers can select appropriate warning levels and they can be driveradjustable. Another strength of using EDM as a CW/CA algorithm is its ability to adapt to different drivers' behaviours. When calibrating errable driver model, model coefficients ( $P$ and $\bar{P}$ in Equations (2) and (3)) are obtained by fitting a set of driving data, which can be customised with individual driving data. Therefore, the errable driver model can be updated in real time for better customisation to the driver. Conventional $\mathrm{CW} / \mathrm{CA}$ systems tuned under
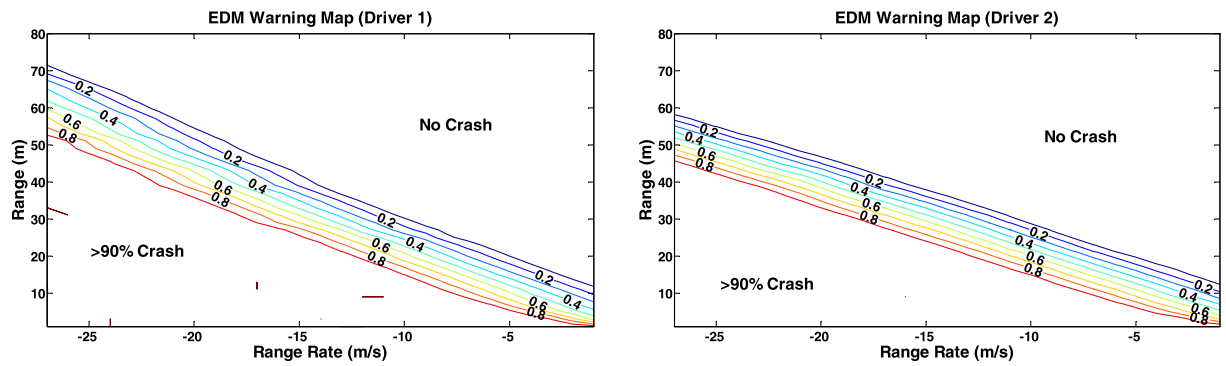

Figure 9. EDM warning map based on the behaviour of two different drivers. 
open-loop were frequently found to work unsatisfactorily with human-in-the-loop. If updated in real time, the obtained $\mathrm{CW} / \mathrm{CA}$ algorithm has the potential to achieve a much higher level of false positives and false negatives, which are critical deficiencies for the commercialisation of $\mathrm{CW} / \mathrm{CA}$ algorithms.

\section{Conclusion}

A humanised errable car-following driver model was used to evaluate the performance of several CW/CA algorithms. This errable driver model emulates human driver and can generate both nominal (error-free) and devious (with error) behaviours. A large-scale naturalistic driving database was used for developing and validating this errable car-following driver model. Three error-inducing behaviours were implemented in this model, human perceptual limitation, time delay and distraction due to non-driving tasks. By combining three error-inducing behaviours, rear-end collision with the lead vehicle occur and at a probability similar to what was reported in traffic accident statistics. This ability of emulating rear-end collision behaviour was useful to evaluate the performance of $\mathrm{CW} / \mathrm{CA}$ algorithms - several of which were shown in this paper and the evaluation results are summarised in this paper.

Subsequently, a new CW/CA algorithm is suggested based on the errable driver model. The driver was used to predict the probability of crash, based on which warning or braking action can be issued. This algorithm was tuned with realistic human driving data. The developed $\mathrm{CW} / \mathrm{CA}$ algorithm has the potential to be tuned in real time to adapt to individual drivers. Efficient computation and actual performance of this $\mathrm{CW} / \mathrm{CA}$ algorithm have not been demonstrated and they are our current research focus.

\section{References}

[1] National Highway Traffic Safety Admission (NHTSA), Traffic Safety Facts 2005: A Compilation of Motor Vehicle Crash Data From Facility Analysis Reporting System and the General Estimates System, National Center for Statistics and Analysis, US Department of Transportation, Washington, DC, 2005.

[2] Army Aviation Board Fort Rucker, Proposed Military Characteristics for Collision Warning Device, 1957.

[3] J.L. Jenkins, An Airborne Collision-Warning Device, Rand Corp Santa Monica CA, 1957.

[4] J.J. Gibson and L.E. Crooks, A theoretical field-analysis of automobile-driving, Am. J. Psychol. 51(3) (1938), pp. 453-471.

[5] H. Kawashima, Integrated system of navigation and communication in Japan, IFAC/IFIP/IFORS Symposium, 1989.

[6] J.K. Pollard, Evaluation of the vehicle radar safety systems' Rashid radar safety brake collision warning system, Washington, DC, February, 1988, DOT TSC HS 802, PM 882.

[7] Z. Parsehhian, Field evaluation of a Nissan laser collision avoidance system, NHTSA Report, Washington, DC, January, 1989, DOT HS 808375.

[8] W.A. Jr. Leasure, The importance of crash problem analysis in defining NHTSA's IVHS program, Proceedings of the IVHS America 1992 Annual Meeting, 1992, pp. 727-732.

[9] R.R. Knipling, D.L. Hendricks, J.S. Koziol, and J.C. Allen, A front-end analysis of rear-end crashes, Proceedings of the IVHS America 1992 Annual Meeting, 1992, pp. 733-745.

[10] R.R. Knipling, M. Mironer, D.L. Hendricks, L. Tijerina, J. Everson, J.C. Allen, and C. Wilson, Assessment of IVHS countermeasures for collision avoidance: Rear-end crashes, NHTSA Report, Washington, DC, May, 1993, DOT HS 807995.

[11] A.L. Burgett, A. Carter, R.J. Miller, W.G. Najan, and D.L. Smith, A collision warning algorithm for rear-end collisions, 16th International Technical Conference on Enhanced Safety of Vehicles Abstracts, 98-S2-P-3 1, Washington, DC, May, 1998.

[12] R. Kiefer, D. LeBlanc, M. Palmer, J. Salinger, and R. Deering, Development and validation of functional definitions and evaluation procedures for collision warning/avoidance system, NHTSA Technical Report, 1999.

[13] A. Doi, T. Butsuen, T. Niibe, T. Takeshi, Y. Yamamoto, and H. Seni, Development of a rear-end collision avoidance system with automatic brake control, JSAE Rev. 15 (1994), pp. 335-340.

[14] S.J. Brunson, E.M. Kyle, N.C. Phamdo, and G.R. Preziotti, Alert algorithm development program NHTSA rear end collision alert algorithm, NHTSA Technical Report, 2002. 
[15] Y. Zhang and E.K. Antonsson, A new assessment measure for collision avoidance systems, Proceedings of the IEEE ITCS, Toronto, Ontario, 2006, pp. 968-975.

[16] J.J. Gibson, The Ecological Approach to Visual Perception, Houghton Mifflin, Boston, 1987.

[17] D.N. Lee, A theory of visual control of braking based on information about time-to-collision, Perception, 5(4) (1976), pp. 437-459.

[18] K. Lee and H. Peng, Evaluation of automotive forward collision warning and collision avoidance algorithm, Veh. Syst. Dyn. 43(10) (2005), pp. 735-751.

[19] S. Hirst and R. Graham, The format and perception of collision warnings, in Ergonomics and Safety of Intelligent Driver Interfaces, L. Erlbaum Associates Inc., 1997, pp. 197-203.

[20] R. Miler and Q. Huang, An adaptive peer-to-peer collision warning system, in Vehicular Technology Conference, Vol. 1, 2002, pp. 317-321.

[21] D. LeBlanc, Road departure crash warning system field operational test: Methodology and results, Vol. 1, Transportation Research Institute (UMTRI), University of Michigan, Ann Arbor, MI, DTFH61-01-X-0053, 2006.

[22] H.-H. Yang and H. Peng, Development of an errorable car-following driver model, Veh. Syst. Dyn. 48(6) (2010), pp. 751-773.

[23] L.A. Pipes, An operational analysis of traffic dynamics, J. Appl. Phys. 24 (1953), pp. 271-281.

[24] J. Tyler Jr, The characteristic of model-following systems as synthesized by optimal control, IEEE Trans. Automat. Control 9(4) (1964), pp. 485-498.

[25] M. Bando, K. Hasebe, K. Nakanishi, and A. Nakayama, Phenomenological study of dynamical model of traffic flow, J. Phys. I France, 5 (1995), pp. 1389-1399.

[26] D.C. Gazis, R. Herman, and R.W. Rothery, Nonlinear follow-the-leader models of traffic flow, Oper. Res. 9(4) (1961), pp. 545-567.

[27] G.F. Newell, Nonlinear effects in the dynamics of car following, Oper. Res. 9(2) (1961), pp. $209-229$.

[28] P.G. Gipps, Behavioral car-following model for computer simulation, Transport. Res., 15B(2) (1981), pp. 105-111.

[29] R. Ervin, J. Sayer, D. LeBlanc, S. Bogard, M. Mefford, Z. Hagan, M. Bareket, and C. Winkler, Automotive collision avoidance system field operational test report, DOT HS 809 900, NHTSA, August 2005.

[30] J.L. Campbell and C.M. Richard, Crash warning system interfaces: Human factors insights and lessons learned, DOT HS 810 697, NHTSA, January 2007.

[31] W.G. Najm and J.D. Smith, Development of crash imminent test scenarios for integrated vehicle safety systems, DOT HS 810 757, NHTSA, April 2007.

[32] L. Yang and J.H. Yang, Development of a performance-based approach for a rear-end collision warning and avoidance system for automobiles, IEEE, 2003.

[33] Y. Fujita, Y. Akuzawa, and M. Sato, Radar brake system, Proceedings of Annual Meeting of IT'S America, March, 1995, pp. 95-101.

[34] K. Lee, Longitudinal driver model and collision warning and avoidance algorithm based on driver databases, Ph.D. diss., University of Michigan, 2004. 